\title{
Biokinetic Modeling and Dosimetry for Optimizing Intraperitoneal Radioimmunotherapy of Ovarian Cancer Microtumors
}

\author{
Stig Palm ${ }^{1}$, Tom Bäck ${ }^{1}$, Börje Haraldsson ${ }^{2}$, Lars Jacobsson $^{1}$, Sture Lindegren ${ }^{1}$, and Per Albertsson ${ }^{3}$ \\ ${ }^{1}$ Department of Radiation Physics, Institute for Clinical Sciences, Sahlgrenska Academy at University of Gothenburg, Gothenburg, \\ Sweden; ${ }^{2}$ Department of Clinical and Molecular Medicine, Institute of Medicine, Sahlgrenska Academy at University of Gothenburg, \\ Gothenburg, Sweden; and ${ }^{3}$ Department of Oncology, Institute for Clinical Sciences, Sahlgrenska Academy at University of \\ Gothenburg, Gothenburg, Sweden
}

\begin{abstract}
A biokinetic model was constructed to evaluate and optimize various intraperitoneal radioimmunotherapies for micrometastatic tumors. The model was used to calculate the absorbed dose to both anticipated microtumors and critical healthy organs and demonstrated how intraperitoneal targeted radiotherapy can be optimized to maximize the ratio between them. Methods: The various transport mechanisms responsible for the biokinetics of intraperitoneally infused radiolabeled monoclonal antibodies (mAbs) were modeled using a software package. Data from the literature were complemented by pharmacokinetic data derived from our clinical phase I study to set parameter values. Results using the $\beta$-emitters ${ }^{188} \mathrm{Re},{ }^{177} \mathrm{Lu}$, and ${ }^{90} \mathrm{Y}$ and the $\mathrm{a}$-emitters ${ }^{211} \mathrm{At},{ }^{213} \mathrm{Bi}$, and ${ }^{212} \mathrm{~Pb}$ were compared. The effects of improving the specific activity, prolonging residence time by introducing an osmotic agent, and varying the activity concentration of the infused agent were investigated. Results: According to the model, a 1.7-L infused saline volume will decrease by $0.3 \mathrm{~mL} / \mathrm{min}$ because of lymphatic drainage and by $0.7 \mathrm{~mL} / \mathrm{min}$ because of the transcapillary convective component. The addition of an osmotic agent serves to lower the radiation dose to the bone marrow. Clinically relevant radioactivity concentrations of $\alpha-$ and $\beta$-emitters bound to mAbs were compared. For a-emitters, microtumors receive high doses (>20 Gy or $100 \mathrm{~Sv}$ [relative biological effect $=5]$ ). Since most of the tumor dose originates from cell-bound radionuclides, an increase in the specific activity would further increase the tumor dose without affecting the dose to peritoneal fluid or bone marrow. For $\beta$-emitters, tumors will receive almost entirely nonspecific irradiation. The dose from cell-bound radiolabeled mAbs will be negligible by comparison. For the long-lived ${ }^{90} \mathrm{Y}$, tumor doses are expected to be low at the maximum activity concentration delivered in clinical studies. Conclusion: According to the presented model, a-emitters are needed to achieve radiation doses high enough to eradicate microscopic tumors.
\end{abstract}

Key Words: radioimmunotherapy; targeted alpha therapy; alphaemitters; ovarian cancer; intraperitoneal therapy

J Nucl Med 2016; 57:594-600

DOI: $10.2967 /$ jnumed.115.167825

Received Oct. 5, 2015; revision accepted Dec. 24, 2015.

For correspondence or reprints contact: Stig Palm, Department of Radiation Physics, University of Gothenburg, Gula Stråket 2B, SE-413 45 Gothenburg, Sweden.

E-mail: stig.palm@gu.se

Published online Jan. 14, 2016.

COPYRIGHT (C 2016 by the Society of Nuclear Medicine and Molecular Imaging, Inc.
A

diagnosis, ovarian cancer has often spread within the peritoneum. Treatment with advanced surgery and consolidated chemotherapy can appear successful, as many patients are declared tumor-free after a second laparoscopy. However, most of these patients will relapse and eventually die. To increase treatment success, adjuvant or consolidating therapies involving radionuclides have been attempted. Intraperitoneal radioimmunotherapy has the potential to irradiate micro- or subclinical tumors that have spread within the peritoneum. Many promising experiments have been performed with $\beta$-emitting radionuclides. Although a multicenter phase III clinical trial of adjuvant ${ }^{90}$ Y-monoclonal antibody $(\mathrm{mAb})$ did not show any survival benefit (1), various $\beta$-emitting radionuclides have shown other promising effects on minimal tumor growth, such as decreased tumor size at repeat operation (2), complete remission at third-look evaluation (3), and prolonged time to intraperitoneal recurrence (4).

The high-linear-energy transfer and short range of $\alpha$-particles (50$100 \mu \mathrm{m})$ facilitates more concentrated irradiation of microscopic tumors. Various $\alpha$-emitters have thus been evaluated for several cancer types (5). Our group used preclinical experiments to study the therapeutic effect and toxicity of the $\alpha$-emitters ${ }^{211} \mathrm{At}$ and ${ }^{213} \mathrm{Bi}$ for intraperitoneal treatment of microscopic tumors and found high tumor doses consistent with possible cure (6-8). The promising results led to the initiation and completion of a phase I clinical study of intraperitoneally infused ${ }^{211} \mathrm{At}-\mathrm{MX} 35 \mathrm{~F}\left(\mathrm{ab}^{\prime}\right)_{2}$ (9). Patient kinetic studies showed that for activity amounts that could be therapeutic, normaltissue radiation doses were low to moderate and there was no acute toxicity. However, the estimated long-term risk is not negligible (10). It is therefore important to optimize therapy in order to maximize the ratio between dose to microtumors and dose to healthy tissues.

The aim of the current work was to build a physiology-based biokinetic model of the transport of intraperitoneally infused antibodies that describes distribution to healthy tissues and binding to microtumors of various sizes. Together with dosimetry, the model should predict the dose to both tumors and critical healthy tissues for various radionuclides and infused solutions. Ideally, the model would explain the results of previous therapies involving both $\beta$ - and $\alpha$-emitters and could be used to guide and optimize future intraperitoneal radioimmunotherapies.

\section{MATERIALS AND METHODS \\ Compartmental Modeling}

Most relevant transport mechanisms (Fig. 1) were simulated using the software package STELLA (ISEE Systems, Inc.). The resulting 


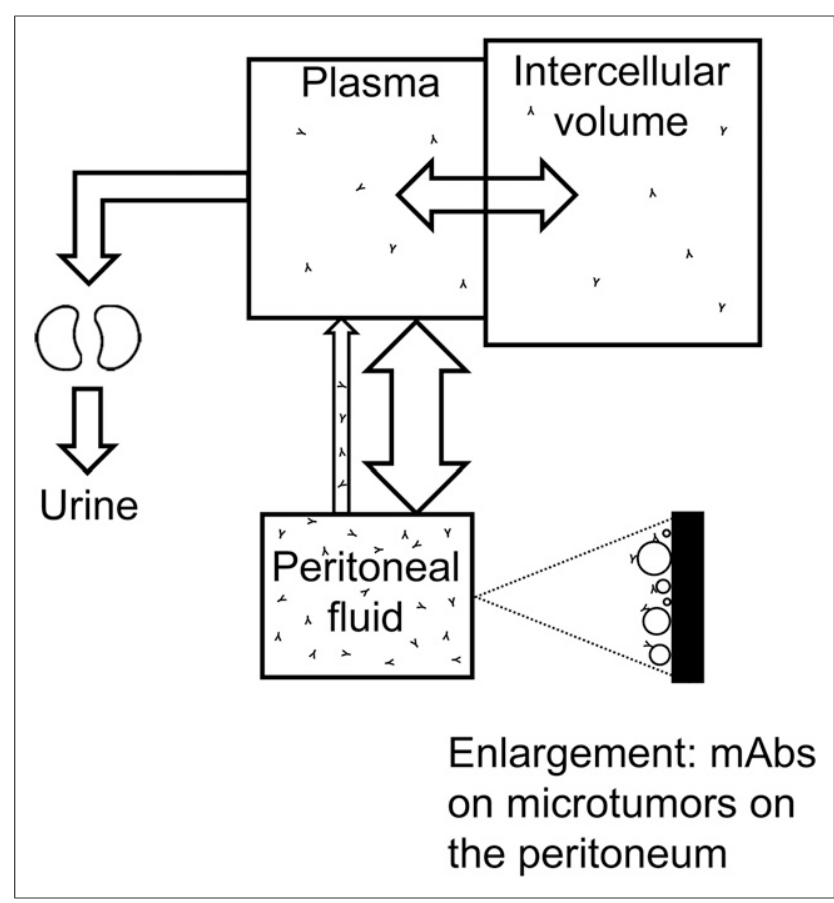

FIGURE 1. Schematic illustration of the main transports included in model. Unidirectional arrow from peritoneal fluid symbolizes lymphatic absorption, which includes mAb transport. Two-headed arrow between peritoneal fluid and plasma reflects transcapillary absorption.

time-dependent biodistribution was then used as input for dosimetry. At the time of intraperitoneal infusion, all radionuclides were assumed to be bound to antibodies. We further assumed that the radioimmunoconjugate was stable within the peritoneum and only slowly degraded in the circulation. To construct the model, we adopted a prior model (7) for estimates of antibody binding to cells and added elements that described transport of antibodies from the peritoneal cavity to the circulation.

\section{Model Parameters}

The uptake kinetics of microtumors were based on in vitro data generated using the cell line NIH:OVCAR-3 and were determined by the association constant, the number of available antigens on the cell surface, and the concentration of mAbs in the peritoneal fluid. Because the in vitro data indicated a negligibly small dissociation constant, this parameter was set at 0 in the model. The radiotherapeutic agent was expected to be infused intraperitoneally in a $1.7-\mathrm{L}$ volume, the average used for patients enrolled in our clinical study (10). For modeling the peritoneal fluid transport, we expected two types of simultaneous absorption: lymphatic and transcapillary.

Lymphatic Absorption. Removal of antibody from the intraperitoneal fluid was assumed to be entirely due to direct absorption by the diaphragmatic lymphatics. A constant rate of this absorption was used as a free parameter in the model. A value of $0.3 \mathrm{~mL} / \mathrm{min}$ yielded model results that best fitted the plasma concentrations of the patients enrolled in our previous trial. Because this value also agreed with what was found in the literature (11), it was used in the model.

Transcapillary Absorption. For infused saline, additional absorption of water due to tissue in contact with the peritoneal fluid was set at $0.7 \mathrm{~mL} / \mathrm{min}$ to match an expected total absorption rate of $1 \mathrm{~mL} / \mathrm{min}$ (11). A residual peritoneal fluid volume of $200 \mathrm{~mL}$ was assumed, representing the level of intraperitoneal fluid remaining after about $25 \mathrm{~h}$. At that time, the flow in the model was instantaneously reversed to match the constant rate of absorption by diaphragmatic lymphatics, that is, $0.3 \mathrm{~mL} / \mathrm{min}$.

For an infused icodextrin solution, the flow rate into the peritoneum was assumed to be proportional to the icodextrin concentration. The value of the proportionality constant was set so that the model results fitted the mean peritoneal fluid concentrations of the patients enrolled in our clinical study. The resulting modeled flow rate varied from 3.2 to $1.5 \mathrm{~mL} / \mathrm{min}$ in the first $24 \mathrm{~h}$, which agreed reasonably well with the 4-h data published for icodextrin (12). Our patients' peritoneal fluid was emptied $24 \mathrm{~h}$ after infusion, but this procedure did not alter the main results of the current work.

The modeling further involved a range of parameter settings that were either drawn from literature (11-14) or based on our own clinical experience. Additional settings involved free parameters that were set so that the results of the model would match the time-dependent plasma and intraperitoneal fluid concentrations of the patients enrolled in our clinical trial. The parameters are summarized in Table 1.

\section{Concentration of mAbs in Plasma and Intercellular Volume}

We expected some delay for antibodies that had departed from the peritoneal cavity before they appeared in plasma. This delay was modeled, with a randomly drawn transit time (normal distribution; mean, $5.0 \mathrm{~h} ; \mathrm{SD}, 6.0 \mathrm{~h}$ ) being assigned for each small amount of departed antibodies, that is, those that departed during the last $0.01 \mathrm{~h}$. Once the antibodies reached the circulation, we assumed that they were instantaneously distributed throughout the plasma volume, constituting $3.6 \%$ of the body weight (15). A distribution volume, fixed at $9.1 \%$ (16) of the body weight, was simulated.

The subsequent 2-way kinetics for molecules transported in and between plasma and the intercellular volume were then modeled using a transport rate of $6.5 \% / \mathrm{h}$ from plasma, as reported for radiolabeled albumin (17). Use of the same transport rate for intercellular volume to plasma provided model results that were a good fit to the patients' measured plasma concentrations.

\section{Dosimetry}

After the number of radionuclide atoms bound to a single cell was determined, the MIRD-cell application (18) was used for tumor dosimetry. Absorbed dose and equivalent dose were used to predict the radiation effect for all evaluated radionuclides even though a detailed microdosimetric evaluation of the $\alpha$-emitters, including the number of events in individual cell nuclei of the microtumors, would likely be a better predictor of effect. Three microtumor geometries, spheres with radii of 9,30 , and $50 \mu \mathrm{m}$, were selected on the basis of preclinical findings from a relevant tumor model (6). A tumor was simulated as a homogeneous tissue-equivalent sphere of $1 \mathrm{~g} / \mathrm{cm}^{3}$. The sphere surface was covered with antigens of a density of $688 / \mu \mathrm{m}^{2}$, equal to that for a single cell. A single point on the surface of the tumor sphere was attached to a flat plane, simulating the peritoneum. It was assumed that there were no neighboring tumors contributing to crossfire. The cell-bound radiolabeled mAbs were distributed on the surface of the tumor sphere where the decays were simulated to occur. The free radiolabeled $\mathrm{mAbs}$ in the surrounding fluid were simulated as randomly distributed. Because no radionuclides were simulated outside the peritoneal plane, the half-space surface geometry allowed the dose contribution to the tumor sphere from radiolabeled mAbs in the peritoneal fluid to be estimated by assigning half the intraperitoneal fluid electron equilibrium dose to the tumor. The dose contribution to the tumor sphere from free $\alpha$-emitting radiolabeled mAbs in the peritoneal fluid was determined using an in-house-developed Monte Carlo program (19), because MIRD-cell does not support this irradiation geometry.

The radiolabeled $\mathrm{mAb}$ concentration in plasma will directly translate to bone marrow dose. The time-dependent concentration in red 
TABLE 1

Summary of Model Parameters

\begin{tabular}{|c|c|c|c|}
\hline Parameter & Amount/rate & Comments & Reference \\
\hline \multicolumn{4}{|l|}{ Fluid } \\
\hline Plasma & $2.3 \mathrm{~L}$ & $36 \mathrm{~mL} / \mathrm{kg}$ of body weight & $(15)$ \\
\hline Distribution volume in tissue & $5.9 \mathrm{~L}$ & $91 \mathrm{~mL} / \mathrm{kg}$ of body weight & $(16)$ \\
\hline Administered intraperitoneal fluid & $1.7 \mathrm{~L}$ & & \\
\hline Residual intraperitoneal fluid & $0.2 \mathrm{~L}$ & & \\
\hline \multicolumn{4}{|l|}{ Intraperitoneal fluid transport } \\
\hline $\begin{array}{l}\text { Lymphatic drainage (intraperitoneal } \\
\text { fluid } \Rightarrow \text { plasma) }\end{array}$ & $0.3 \mathrm{~mL} / \mathrm{min}$ & Mean delay, 5 h ( \pm 6 h; SD) & Model fit \\
\hline $\begin{array}{l}\text { Water reabsorption (intraperitoneal } \\
\text { fluid } \Rightarrow \text { plasma) }\end{array}$ & $0.7 \mathrm{~mL} / \mathrm{min}$ & Peritoneal fluid $>200 \mathrm{~mL}$ & Model fit \\
\hline $\begin{array}{l}\text { Water inflow at equilibrium } \\
\text { (intraperitoneal fluid } \Leftarrow \text { plasma) }\end{array}$ & $0.3 \mathrm{~mL} / \mathrm{min}$ & Peritoneal fluid $=200 \mathrm{~mL}$ & Model fit \\
\hline $\begin{array}{l}\text { Water inflow; osmotic effect } \\
\quad \text { (intraperitoneal fluid } \Leftarrow \text { plasma) }\end{array}$ & $3.1-1.5 \mathrm{~mL} / \mathrm{min}$ & $\begin{array}{l}\text { Proportional to intraperitoneal } \\
\text { icodextran concentration, 0-24 h }\end{array}$ & Model fit \\
\hline \multicolumn{4}{|l|}{ mAb conjugate transfer coefficients } \\
\hline TER (plasma $\Leftrightarrow$ intercellular volume) & $0.065 \mathrm{~h}^{-1}$ & & $(17)$ \\
\hline $\begin{array}{l}\text { Degradation/excretion (plasma } \Rightarrow \\
\text { urine) }\end{array}$ & $0.0096-0.03 h^{-1}$ & Radiolabel-dependent & Model fit \\
\hline \multicolumn{4}{|l|}{ mAb binding parameters } \\
\hline $\begin{array}{l}\text { Association constant (intraperitoneal } \\
\text { fluid } \Rightarrow \text { tumor cell) }\end{array}$ & $44,000 \mathrm{M}^{-1} \mathrm{~s}^{-1}$ & & (6) \\
\hline $\begin{array}{l}\text { Dissociation constant (tumor cell } \Rightarrow \\
\text { intraperitoneal fluid) }\end{array}$ & $0 \mathrm{~s}^{-1}$ & & $(6)$ \\
\hline Number of sites per cell & 700,000 & & $(6)$ \\
\hline $\mathrm{TER}=$ transcapillary escape rate. & & & \\
\hline
\end{tabular}

bone marrow was determined from a fixed ratio of 0.19 (20), although this ratio has been shown to vary with time and among patients (21). The absorbed dose to bone marrow was then calculated by multiplying the cumulated activity by the average $\alpha$ - or $\beta$-particle energy emitted

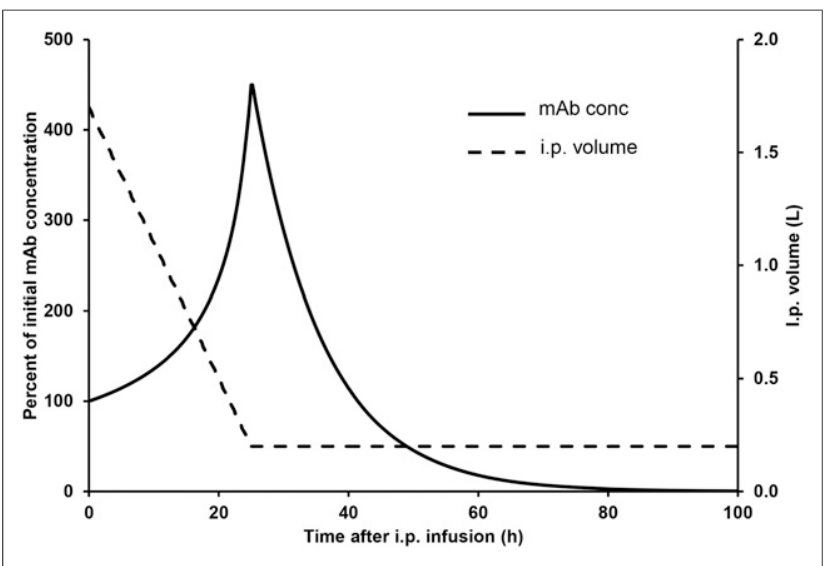

FIGURE 2. Simulated intraperitoneal fluid volume (dashed line) and relative $\mathrm{mAb}$ concentration (solid line) after intraperitoneal infusion of 1.7 $\mathrm{L}$ of saline. Rapid change in mAb concentration is due to reversal of flow when residual intraperitoneal fluid volume of $200 \mathrm{~mL}$ is reached. i.p. = intraperitoneal. per decay. An absorbed fraction of 1 for the $\alpha$-particles and electrons was assumed, whereas the contribution from $\gamma$-particles was considered negligible.

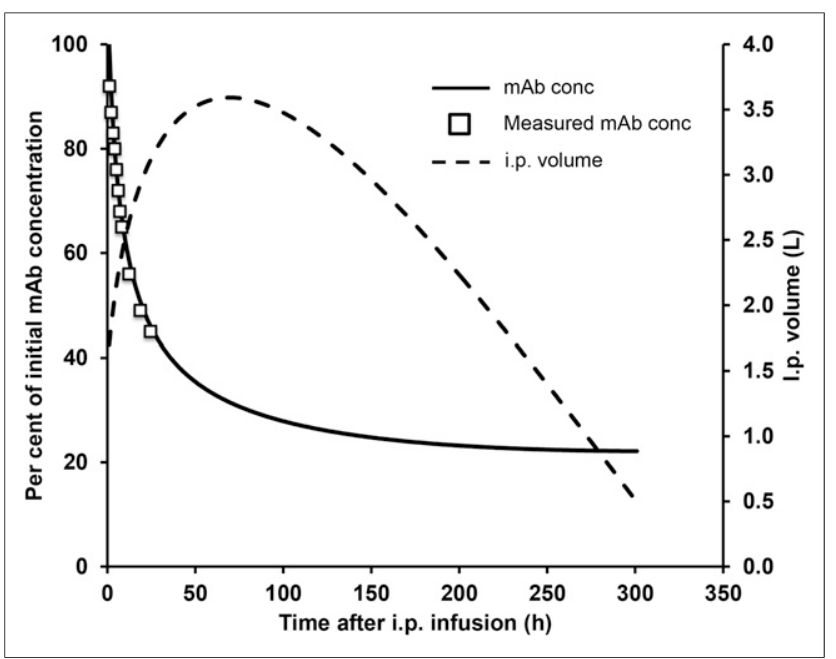

FIGURE 3. Simulated intraperitoneal fluid volume (dashed line) and relative $\mathrm{mAb}$ concentration (solid line) after intraperitoneal infusion of $1.7 \mathrm{~L}$ of $7.5 \%$ icodextrin solution. Measured data from patients' intraperitoneal fluid samples are presented as open squares. i.p. = intraperitoneal. 


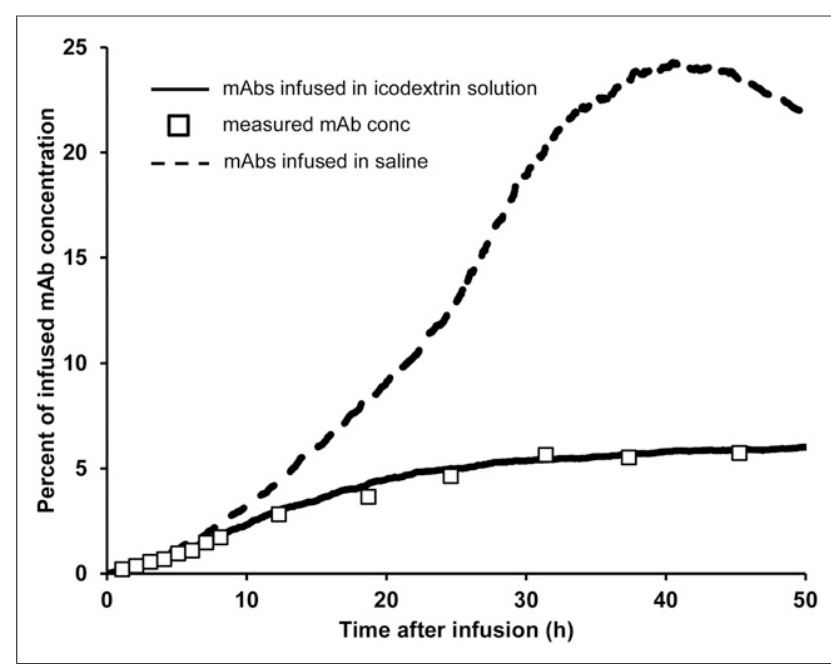

FIGURE 4. Concentration of mAb in plasma after infusion of $1.7 \mathrm{~L}$ of $\mathrm{mAb}$ in saline (dashed line) or $7.5 \%$ icodextrin solution (solid line). Measured samples from patients enrolled in our phase I clinical study are presented as open squares.

\section{RESULTS}

\section{Peritoneal Fluid Volume}

The 1.7- $\mathrm{L}$ infused saline volume decreased by $0.3 \mathrm{~mL} / \mathrm{min}$ because of lymphatic drainage and by $0.7 \mathrm{~mL} / \mathrm{min}$ because of the transcapillary convective component. Because larger molecules diffuse slowly and their passage across capillary walls is restricted, their concentration was initially increased. As the residual fluid volume of $200 \mathrm{~mL}$ was reached, the net influx by the transcapillary component became equal to the constant lymphatic drainage. At that point, the mAb concentration decreased as it was slowly diluted (Fig. 2).

With the addition of the osmotic agent, the transcapillary component was reversed and an initial net influx of water into the peritoneal cavity occurred. Because the osmotic effect gradually decreased, the maximum intraperitoneal fluid volume was reached at about $70 \mathrm{~h}$ after infusion, after which lymphatic drainage caused a net decrease in volume. The initial net influx of fluid resulted in dilution of the mAb. The results agreed with measured data from our patients' intraperitoneal fluid samples (Fig. 3).

\section{Antibody Concentration in Plasma}

Antibodies reached the circulation solely by lymphatic drainage of the intraperitoneal fluid. The mAb concentration in plasma is presented in Figure 4 as the percentage of initial mAb concentration in the $1.7 \mathrm{~L}$ of intraperitoneally infused fluid. The results for saline were within the broad ranges presented in the literature. The higher concentration in plasma seen after an isotonic infusion was due to the higher mAb concentration in intraperitoneal fluid (Fig. 2). The data agreed with measured plasma samples from patients enrolled in our phase I clinical study.

\section{Bone Marrow Dose}

Table 2 lists the estimated bone marrow doses after intraperitoneal infusion of mAbs with various radiolabels and hypertonic infused fluid. Because the concentration of mAbs in plasma is significantly higher for an isotonic intraperitoneal infusion (Fig. 4), the resulting bone marrow dose is higher, illustrating how adding the osmotic agent lowers the radiation dose to bone marrow.

\section{Microtumor Uptake}

The average number of mAbs bound per tumor cell was calculated. Two examples are shown in Figure 5. One is $300 \mathrm{MBq}$ of ${ }^{211} \mathrm{At}-\mathrm{mAb}(0.56 \mathrm{mg})$, with a specific activity translating to 1 of $200 \mathrm{mAbs}$ labeled with an ${ }^{211}$ At atom, intraperitoneally infused in a volume of $1.7 \mathrm{~L}$. The other was $3,000 \mathrm{MBq}$ of ${ }^{213} \mathrm{Bi}-\mathrm{mAb}(0.59 \mathrm{mg})$, which also had a specific activity translating to radiolabeling of 1 of $200 \mathrm{mAbs}$. The small difference in the number of cell-bound mAbs between isotonic and hypertonic infused fluid is shown in Figure 5. The results were used to calculate the average number of ${ }^{211} \mathrm{At}$ or ${ }^{213} \mathrm{Bi}$ atoms per cell and the cumulative number of decays- that is, cumulated activity—per cell.

\section{Microtumor Dosimetry}

Dosimetry was performed for single cells and spheric cell clusters with diameters of 60 and $100 \mu \mathrm{m}$. A relative biological effect of 5 was used to calculate the equivalent dose from $\alpha$-particle irradiation (22). Table 2 lists results for specific activities and activity amounts

TABLE 2

Model Results from Using 1.7 L of Intraperitoneally Infused Radiolabeled mAbs in Osmotic Agent

\begin{tabular}{|c|c|c|c|c|c|c|c|c|c|c|c|}
\hline \multirow[b]{3}{*}{ Nuclide } & \multirow{3}{*}{$\begin{array}{l}\text { Fraction of } \\
\text { mAbs } \\
\text { radiolabeled }\end{array}$} & \multirow{3}{*}{$\begin{array}{l}\text { Administered } \\
\text { activity (MBq) }\end{array}$} & \multirow{3}{*}{$\begin{array}{l}\text { Decays } \\
\text { per } \\
\text { cell }(n)\end{array}$} & \multicolumn{8}{|c|}{ Equivalent dose (Sv) (RBE, 5 for a-particles and 1 for electrons) } \\
\hline & & & & \multirow{2}{*}{$\begin{array}{l}\text { Bone } \\
\text { marrow }\end{array}$} & \multirow{2}{*}{$\begin{array}{l}\text { Peritoneal } \\
\text { fluid }\end{array}$} & \multicolumn{3}{|c|}{ Tumor (from cell-bound mAbs) } & \multicolumn{3}{|c|}{ Tumor (total) } \\
\hline & & & & & & $\mathrm{D}, 18 \mu \mathrm{m}$ & $\mathrm{D}, 60 \mu \mathrm{m}$ & $\mathrm{D}, 100 \mu \mathrm{m}$ & $\mathrm{D}, 18 \mu \mathrm{m}$ & $\mathrm{D}, 60 \mu \mathrm{m}$ & $\mathrm{D}, 100 \mu \mathrm{m}$ \\
\hline${ }^{177} \mathrm{Lu}$ & $1 / 270$ & 3,900 & 2,561 & 0.94 & 17 & 0.43 & 0.34 & 0.30 & 8.9 & 8.8 & 8.8 \\
\hline $90 Y$ & $1 / 270$ & 1,100 & 2,580 & 0.61 & 26 & 0.13 & 0.12 & 0.12 & 13 & 13 & 13 \\
\hline${ }^{188} \mathrm{Re}$ & $1 / 270$ & 6,300 & 2,561 & 1.02 & 68 & 0.22 & 0.18 & 0.17 & 34 & 34 & 34 \\
\hline${ }^{211} \mathrm{At}$ & $1 / 200$ & 300 & 2,602 & 0.14 & 24 & 231 & 278 & 264 & 244 & 292 & 275 \\
\hline${ }^{213} \mathrm{Bi}$ & $1 / 200$ & 3,000 & 953 & 0.02 & 43 & 71 & 80 & 93 & 94 & 104 & 114 \\
\hline${ }^{212} \mathrm{~Pb}$ & $1 / 200$ & 300 & 2,995 & 0.36 & 37 & 244 & 283 & 288 & 264 & 305 & 305 \\
\hline
\end{tabular}



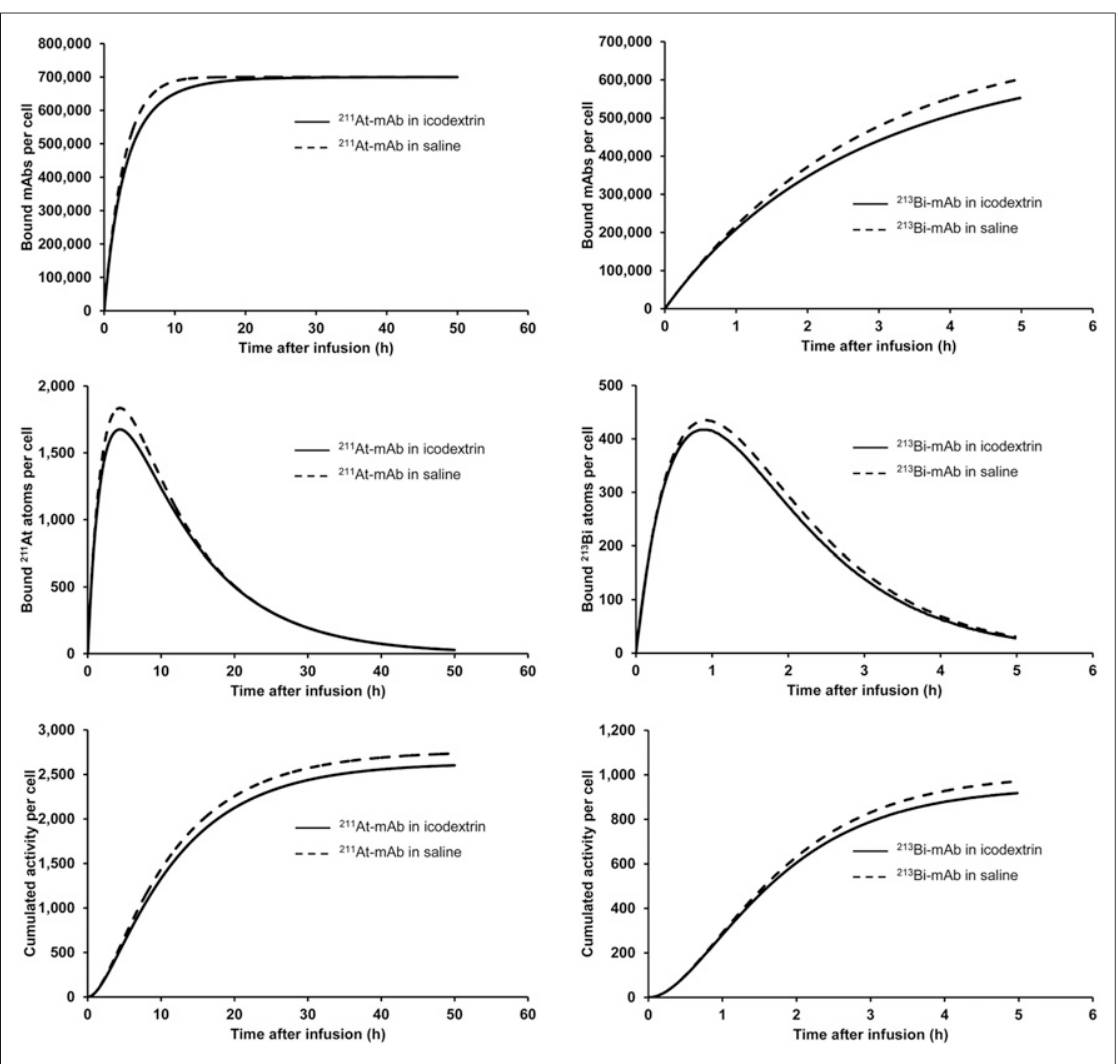

FIGURE 5. Illustration of how simulated cell-binding kinetics of radiolabeled mAbs determines absorbed dose to tumors. Top panels show total number of mAbs bound per cell. Middle panels show expected number of ${ }^{211} \mathrm{At}$ or ${ }^{213} \mathrm{Bi}$ atoms bound per cell at any specific time, that is, nondecay-corrected, assuming 1 of 200 mAbs radiolabeled at time of intraperitoneal infusion. Bottom panels show cumulated number of decays per cell, which translates to absorbed dose. Dashed lines represent results after intraperitoneal infusion of $1.7 \mathrm{~L}$ of saline, whereas solid lines are results for $1.7 \mathrm{~L}$ of $7.5 \%$ icodextrin. Figure also illustrates how use of icodextrin only slightly reduces tumor dose (but results in large decrease in dose to healthy tissues).

that are reasonably achievable today. For the $\alpha$-emitters, microtumors received high doses. Because most of the tumor dose originated from cell-bound radionuclides, an increase in specific activity will further increase the tumor dose without affecting the dose to peritoneal fluid or bone marrow. Tumor doses from unbound radiolabeled mAbs in the surrounding intraperitoneal fluid were close to $50 \%$ (range, $40 \%-$ $60 \%$ ) of the fluid equilibrium dose for those $\alpha$-emitters and tumor sizes that were investigated.

When $\beta$-emitters were used, less than $5 \%$ of the radiation dose to tumors was due to decay on tumor surfaces. The remainder of the dose, that is, more than $95 \%$, was due to irradiation by decay occurring in the surrounding intraperitoneal fluid. For the longer-lived ${ }^{90} \mathrm{Y}$, tumor doses are expected to be low at the maximum activity concentration delivered in clinical studies. For the shorter-lived ${ }^{188} \mathrm{Re}$, unspecific irradiation from the peritoneal fluid would result in tumor doses of $34 \mathrm{~Gy}$ at a tolerable bone marrow dose ( $\sim 1 \mathrm{~Gy})$.

\section{Optimization}

Optimal use of radiation for therapy involves maximizing the ratio between absorbed dose to tumors and absorbed dose to critical healthy organs. Further, for cure, the absorbed dose must be high enough to eradicate the tumors. For $\beta$-emitters, the model showed that the results were best for ${ }^{188} \mathrm{Re}$, the shortest-lived of those evaluated. Specific activity was not important since unbound radiolabeled $\mathrm{mAbs}$ dominated the irradiation.
Optimization for $\alpha$-emitters, for which binding to tumor cells determines the tumor dose, also depends on the half-life of the radionuclide. For treatment with ${ }^{211} \mathrm{At}$, the best gain in tumor dose was achieved by improving the specific activity of the radioimmunoconjugate (Fig. 6). If 1 of $25 \mathrm{mAbs}$ can be radiolabeled, a concentration of approximately $25 \mathrm{MBq} / \mathrm{L}$ is enough to achieve therapeutic tumor doses ( 20 Gy or $100 \mathrm{~Sv}$ [relative biological effect, 5]) for cells with 700,000 antigens and a very low dose to normal tissues, that is, at low risk. A low specific activity cannot be compensated for by using a higher activity concentration, but a higher activity concentration will improve the treatment if the specific activity is high.

\section{DISCUSSION}

In radioimmunotherapy, it is the range of the emitted particle that determines the fraction of total radiation energy absorbed in small volumes such as microtumors. Because the $\beta$-emitters used for intraperitoneal radioimmunotherapy have a relatively long range, on the order of millimeters, the absorbed dose to a cell will remain low even if a large number of radionuclides are bound to the cell surface. For the illustrative cases presented in this work, the absorbed dose from cellbound radionuclides to cell clusters (with diameters $\leq 0.1 \mathrm{~mm}$ ) is negligible in comparison to the dose received from radiolabeled $\mathrm{mAbs}$ in the surrounding intraperitoneal fluid. The total tumor dose is only moderate since the permissible amount of administered radioactivity is strictly limited by the resulting irradiation of critical healthy tissue, particularly bone marrow.

Sparing critical healthy tissues is, according to the presented model, particularly challenging for radionuclides with half-lives greater than about $24 \mathrm{~h}$ because a larger fraction will decay outside the peritoneal cavity. According to our results, the use of shorterlived $\beta$-emitters improves the tumor-to-critical-normal-organ ratios but probably not enough to eradicate all microtumors. The $\beta$-emitters that have been clinically evaluated so far have a relatively long half-life. The restricted administered activity in combination with the long particle ranges results, according to our model, in absorbed doses not near cell sterilization levels for microscopic tumors. However, for macroscopic tumors with diameters of several millimeters, $\beta$-emitters have provided measurable antitumor effects as seen for ${ }^{131} \mathrm{I}(3),{ }^{186} \mathrm{Re}(2)$, and ${ }^{90} \mathrm{Y}(4)$.

$\alpha$-emitters have a short $(50-100 \mu \mathrm{m})$ particle range and highlinear-energy transfer, but their half-lives can differ greatly. A short range in combination with high-linear-energy transfer is key to achieving high radiation doses to intraperitoneal microtumors but also involves irradiation of healthy tissues with uncertain biologic effects, including a long-term risk for secondary cancer that may not be negligible. Minimizing the irradiation of healthy 


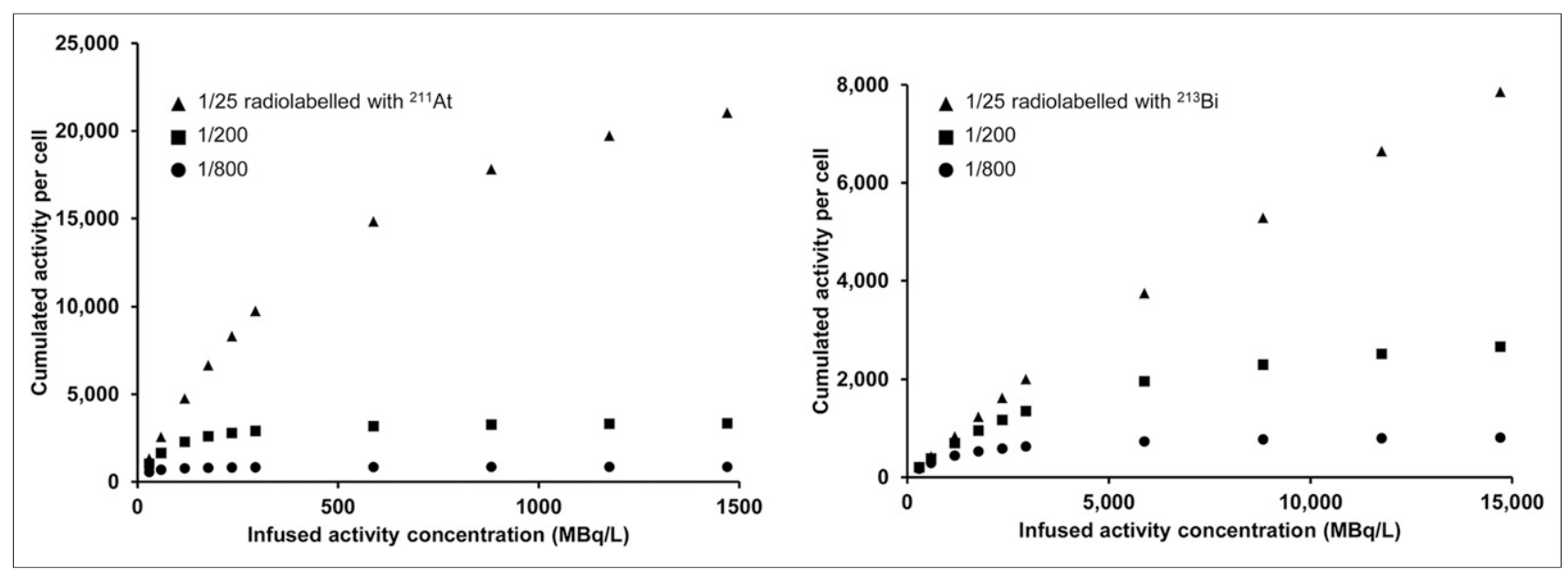

FIGURE 6. Specific activity and intraperitoneally infused activity concentration determine number of radionuclide decays per cell. Specific activity is represented as radiolabeling of 1 of 25,1 of 200 , and 1 of $800 \mathrm{mAbs}$. It is only for high specific activities of radioimmunoconjugate that increase in activity concentration of infused fluid results in significantly higher tumor doses.

tissues is therefore of the utmost importance in an adjuvant setting when long-term carcinogenic risks must be considered (10).

The lymphatic flow and peritoneal fluid concentration of mAbs determine the rate at which they leave the peritoneal cavity. For short-lived radionuclides with a negligible photon contribution, the resulting systemic irradiation is determined by this rate. For longer-lived radionuclides, the lymphatic flow, albeit slow, will have transported almost all radionuclides before they decay in the circulation. Any attempt to modify the rate at which radiolabeled mAbs depart from the peritoneal cavity is thus important only for shorter-lived radionuclides. In our clinical study with ${ }^{211} \mathrm{At}$, we used icodextrin to retain a large volume of intraperitoneal fluid, primarily to guarantee several hours of complete exposure of the peritoneum. Our model showed that the diluting effect also reduced the rate of mAbs entering the circulation, reducing the dose to normal tissues by approximately $50 \%$. The reduction in tumor dose was negligible because the dilution was slow and became significant only after tumor uptake was almost complete.

Absorbed doses to tumor cells depends both on the concentration of radiolabeled antibody within the peritoneum, that is, administered activity and fluid volume, and on specific activity. The specific activity of a radioimmunoconjugate is normally expressed as Bq/g. We chose, instead, to express it as the fraction of mAbs labeled with a radionuclide. The theoretically maximal number of radionuclide atoms bound to the cell membrane depends on this specific activity and is limited by the number of available antigens. Because some atoms decay during the binding process and cell-bound mAbs may be released, the maximum is never fully reached. According to the model results, for a fixed high specific activity, a higher infused ${ }^{211} \mathrm{At}-\mathrm{mAb}$ activity concentration would increase tumor dose through a more rapid binding process. For a fixed low specific activity, increased ${ }^{211}$ At-mAb activity concentrations would increase irradiation of healthy tissues but would only slightly increase tumor dose. To deliver high tumor doses with ${ }^{213} \mathrm{Bi}-\mathrm{mAb}$, cell binding must be rapid, that is, involve high $\mathrm{mAb}$ concentrations, and a large fraction of the mAbs must be labeled with a ${ }^{213} \mathrm{Bi}$ atom, that is, specific activity must be high.

The parameter values in the model were set from literature data or derived from pharmacokinetic data from our clinical phase I study. Thus, the model was constructed to provide perfect agree- ment with measured concentrations of ${ }^{211} \mathrm{At}-\mathrm{mAb}$ in the plasma and peritoneal fluid of these patients. Because this procedure was used to set the free parameters of the model, any single value of these parameters might carry considerable error. However, the accuracy of the general conclusions drawn from the presented results is not affected.

With all feasible optimizations applied, the model predicts the best therapeutic results for ${ }^{211} \mathrm{At}-\mathrm{mAb}$. Good results would also be expected for ${ }^{212} \mathrm{~Pb}-\mathrm{mAb}$ but only if all, instead of the reported $65 \%$ (23), of the radionuclide daughters decay where the parent ${ }^{212} \mathrm{~Pb}$ decay. In addition, higher activity concentrations and specific activities would be needed than those reported (24). Use of short-lived $\alpha$-emitters such as ${ }^{213} \mathrm{Bi}$ requires high activity concentrations to eradicate microtumors. However, the short half-life will reduce normal-tissue irradiation. Excluding the dose to the peritoneum, ${ }^{213} \mathrm{Bi}$ provides a better ratio of dose to tumor relative to normal tissue than does ${ }^{211}$ At. However, because the tolerance dose to the peritoneum is not known, the administered activity concentration of ${ }^{213} \mathrm{Bi}$ might be limited. Finally, if the targeted cells have significantly less antigen expression than used in the model, only ${ }^{211} \mathrm{At}-\mathrm{mAb}$ treatment with very high specific activity would be successful for microtumors.

\section{CONCLUSION}

Through the use of physiologic data, it was possible to construct a model that fit measured radionuclide concentrations in the peritoneal fluid and blood of patients treated intraperitoneally with radiolabeled mAbs. The model is therefore useful for simulation and absorbed dose estimations of therapies with various radiolabeled mAbs.

Targeted $\beta$-emitting therapies have resulted in clinical benefit, but according to the model, $\alpha$-emitters are needed to optimize treatment of microscopic tumors. High-specific-activity ${ }^{211}$ At-mAbs achieve high tumor doses even for cells with a low antigen expression. With a high specific activity, sterilizing tumor doses can be achieved with a low activity concentration that spares normal tissues. Similar results can be achieved with ${ }^{212} \mathrm{~Pb}$ if the radionuclide daughter-mAb complex is stable, or with high-specific-activity ${ }^{213} \mathrm{Bi}-\mathrm{mAb}$, but administration of several gigabecquerels of total activity would be required. 


\section{DISCLOSURE}

The costs of publication of this article were defrayed in part by the payment of page charges. Therefore, and solely to indicate this fact, this article is hereby marked "advertisement" in accordance with 18 USC section 1734. Financial support was provided by the Swedish Research Council, the Swedish Cancer Society, the King Gustav V Jubilee Clinic Research Foundation, and the Regional Agreement on Medical Training and Clinical Research (ALF). No other potential conflict of interest relevant to this article was reported.

\section{REFERENCES}

1. Verheijen RH, Massuger LF, Benigno BB, et al. Phase III trial of intraperitoneal therapy with yttrium-90-labeled HMFG1 murine monoclonal antibody in patients with epithelial ovarian cancer after a surgically defined complete remission. J Clin Oncol. 2006;24:571-578.

2. Jacobs AJ, Fer M, Su FM, et al. A phase I trial of a rhenium 186-labeled monoclonal antibody administered intraperitoneally in ovarian carcinoma: toxicity and clinical response. Obstet Gynecol. 1993;82:586-593.

3. Crippa F, Bolis G, Seregni E, et al. Single-dose intraperitoneal radioimmunotherapy with the murine monoclonal antibody I-131 MOv18: clinical results in patients with minimal residual disease of ovarian cancer. Eur J Cancer. 1995;31A: 686-690.

4. Oei AL, Verheijen RH, Seiden MV, et al. Decreased intraperitoneal disease recurrence in epithelial ovarian cancer patients receiving intraperitoneal consolidation treatment with yttrium-90-labeled murine HMFG1 without improvement in overall survival. Int J Cancer. 2007;120:2710-2714.

5. Elgqvist J, Frost S, Pouget JP, Albertsson P. The potential and hurdles of targeted alpha therapy: clinical trials and beyond. Front Oncol. 2014;3(article 324):1-9.

6. Elgqvist J, Andersson H, Back T, et al. Alpha-radioimmunotherapy of intraperitoneally growing OVCAR-3 tumors of variable dimensions: outcome related to measured tumor size and mean absorbed dose. J Nucl Med. 2006;47:1342-1350.

7. Elgqvist J, Andersson H, Back T, et al. Therapeutic efficacy and tumor dose estimations in radioimmunotherapy of intraperitoneally growing OVCAR-3 cells in nude mice with ${ }^{211}$ At-labeled monoclonal antibody MX35. J Nucl Med. 2005;46:1907-1915.

8. Chouin N, Lindegren S, Frost SH, et al. Ex vivo activity quantification in micrometastases at the cellular scale using the alpha-camera technique. J Nucl Med. 2013;54:1347-1353.
9. Andersson H, Cederkrantz E, Back T, et al. Intraperitoneal alpha-particle radioimmunotherapy of ovarian cancer patients: pharmacokinetics and dosimetry of ${ }^{211}$ At-MX35 F(ab')2: a phase I study. J Nucl Med. 2009;50:1153-1160.

10. Cederkrantz E, Andersson H, Bernhardt P, et al. Absorbed doses and risk estimates of ${ }^{211}$ At-MX35 $\mathrm{F}\left(\mathrm{ab}^{\prime}\right) 2$ in intraperitoneal therapy of ovarian cancer patients. Int J Radiat Oncol Biol Phys. 2015;93:569-576.

11. Waniewski J. Peritoneal fluid transport: mechanisms, pathways, methods of assessment. Arch Med Res. 2013;44:576-583.

12. Moberly JB, Mujais S, Gehr T, et al. Pharmacokinetics of icodextrin in peritoneal dialysis patients. Kidney Int Suppl. 2002;81(suppl):S23-S33.

13. Krediet RT, Lindholm B, Rippe B. Pathophysiology of peritoneal membrane failure. Perit Dial Int. 2000;20(suppl 4):S22-S42.

14. Stachowska-Pietka J, Waniewski J, Flessner MF, Lindholm B. Distributed model of peritoneal fluid absorption. Am J Physiol Heart Circ Physiol. 2006;291: H1862-H1874.

15. Nadler SB, Hidalgo JH, Bloch T. Prediction of blood volume in normal human adults. Surgery. 1962;51:224-232.

16. Garzone PD, Atkinson AJ Jr. In search of physiologically based distribution volume estimates for macromolecules. Clin Pharmacol Ther. 2012;92:419_ 421.

17. Galatius S, Bent-Hansen L, Wroblewski H, Sorensen VB, Norgaard T, Kastrup J. Plasma disappearance of albumin and impact of capillary thickness in idiopathic dilated cardiomyopathy and after heart transplantation. Circulation. 2000;102: 319-325.

18. Vaziri B, Wu H, Dhawan AP, Du P, Howell RW. MIRD pamphlet no. 25: MIRDcell V2.0 software tool for dosimetric analysis of biologic response of multicellular populations. J Nucl Med. 2014;55:1557-1564.

19. Palm S, Humm JL, Rundqvist R, Jacobsson L. Microdosimetry of astatine-211 single-cell irradiation: role of daughter polonium-211 diffusion. Med Phys. 2004;31:218-225

20. Sgouros G. Bone marrow dosimetry for radioimmunotherapy: theoretical considerations. J Nucl Med. 1993;34:689-694.

21. Schwartz J, Humm JL, Divgi CR, Larson SM, O'Donoghue JA. Bone marrow dosimetry using ${ }^{124}$ I-PET. J Nucl Med. 2012;53:615-621.

22. Feinendegen LE, McClure JJ. Meeting report: alpha-emitters for medical therapyworkshop of the United States Department of Energy; Denver, Colorado; May 3031, 1996. Radiat Res. 1997;148:195-201.

23. Baidoo KE, Milenic DE, Brechbiel MW. Methodology for labeling proteins and peptides with lead-212 ( $\left.{ }^{212} \mathrm{~Pb}\right)$. Nucl Med Biol. 2013;40:592-599.

24. Milenic DE, Baidoo KE, Brechbiel MW. Bench to bedside: stability studies of GMP produced trastuzumab-TCMC in support of a clinical trial. Pharmaceuticals (Basel). 2015;8:435-454. 